\title{
Discontinuation of Disease-modifying Treatments in Relapsing-remitting Multiple Sclerosis - A Systematic Literature Review of Observational Studies
}

\author{
Gerard T Harty, ${ }^{1}$ Schiffon L Wong, ${ }^{1}$ Michael Tang ${ }^{2}$ and Sangeeta Budhia ${ }^{2}$ \\ 1. EMD Serono, Inc., Billerica, MA, USA; 2. PAREXEL International, London, UK
}

DOl: https://doi.org/10.17925/ENR.2019.14.1.36

ntroduction: Disease-modifying treatments (DMTs) for relapsing-remitting multiple sclerosis (RRMS) are often prescribed over long time periods and their effectiveness may be limited due to high treatment discontinuation rates. A systematic literature review (SLR) was conducted to summarise the DMT discontinuation data in adults with RRMS and describe the reasons for DMT discontinuation when available. Methods: The SLR was performed according to the Preferred Reporting Items for Systematic Reviews and Meta-Analyses (PRISMA) statement. English-language publications of approved RRMS DMTs were searched from inception until December 2016 in the following databases: EMBASE, MEDLINE, MEDLINE In-process, and Cochrane Central Register of Controlled Trials. Results: A total of 82 studies met the initial pre-specified criteria of the SLR. After applying additional selection criteria, 24 studies (from 37 publications) remained that reported discontinuation rates as a key objective, with the majority (14 studies) evaluating only injectable DMTs. Data were reported in a qualitative/narrative manner. Conclusion: Few studies specifically investigated treatment discontinuation in RRMS. Particularly with the introduction of new treatment options, this is a critical consideration when selecting DMTs. The most common reasons for discontinuing treatment were adverse events, lack of efficacy, and pregnancy, highlighting the importance of the risk/benefit profile when determining the appropriate therapy for RRMS.

\section{Keywords}

Relapsing-remitting multiple sclerosis, RRMS, treatment discontinuation, disease-modifying therapy, DMT, systematic review

Disclosures: Gerard T Harty and Schiffon L Wong are employees of EMD Serono. Michael Tang and Sangeeta Budhia are employed by PAREXEL and provided consultancy services funded by EMD Serono Research and Development, a subsidiary of Merck KGaA.

Review Process: Double-blind peer review.

Acknowledgements: Assistance with the drafting of the manuscript was provided by Jo Whelan of Textpharm Ltd and Jason Allaire, PhD, of Generativity Solutions Group, Durham, North Carolina, USA. Assistance with conducting the literature review was provided by Kashif Siddiqui and Inderpreet Singh of PAREXEL. Assistance was funded by EMD Serono.

Compliance with Ethics: This study involves a review of the literature and did not involve any studies with human or animal subjects performed by any of the authors.

Authorship: All named authors meet the International Committee of Medical Journal Editors (ICMJE) criteria for authorship of this manuscript, take responsibility for the integrity of the work as a whole, and have given final approval for the version to be published.

Received: 21 May 2019

Accepted: 5 June 2019

Citation: European Neurological Review, 2019;14(1):36-43

Corresponding Author: Gerard T Harty, EMD

Serono, Inc, 45A Middlesex Turnpike, Billerica, MA 01821, USA. E: gerard.harty@emdserono.com

Support: This study and the publication of this article was supported by EMD Serono.
Multiple sclerosis (MS) is a chronic inflammatory, demyelinating disorder of the central nervous system that is characterised by episodes of neurological dysfunction and eventually, neurodegeneration and the progressive accumulation of disability. ${ }^{12}$ Approximately $85 \%$ of patients with MS initially present with relapsing-remitting MS (RRMS), which is characterised by periodic acute exacerbations of disease activity (relapses) followed by periods of remission. ${ }^{1-5}$ Globally, an estimated 2.3 million people were living with MS in 2013, with 1.23 million disability-adjusted life years (DALYS) attributed to MS in 2015. ${ }^{\circ}$

There is currently no cure for MS but there are a number of disease-modifying treatments (DMTS) that can help control the condition and delay disability progression, particularly if treatment is initiated early in the disease course. ${ }^{7-9}$ The recommendation for early initiation of treatment, combined with the chronic and progressive nature of MS and the early age of onset (typically between 20-30 years of age), often means DMTs are prescribed over a long period of time. However, their effectiveness may be limited due to poor rates of adherence and persistence, and high rates of treatment discontinuation. Discontinuation is associated with negative clinical outcomes such as increased risk of relapse, disease progression, and even rebound disease activity. ${ }^{10-14}$ In addition, poor adherence may result in greater healthcare resource utilisation and cost.9.15-17

Rates of treatment discontinuation and persistence vary widely across studies of RRMS. ${ }^{9,15-19}$ Furthermore, reasons for discontinuing DMTs are not always well documented in the literature, with clinical rationale and context often missing from the information reported. A clear understanding of patterns of treatment discontinuation and switching among patients with RRMS receiving licensed doses of DMTs, and the reasons behind these decisions, is important given the potential impact of such factors on clinical practice. A systematic literature review (SLR) of studies reporting real world patterns of treatment discontinuations (excluding randomised controlled trials [RCTs] and case studies/reports) was conducted with the primary objective of summarising DMT discontinuation data in adults with RRMS. A secondary objective was to review and describe the reasons for DMT discontinuation when available.

\section{Methods}

Literature review

A SLR was performed in accordance with the Preferred Reporting Items for Systematic Reviews and Meta-Analyses (PRISMA) statement (see Supplementary Table 1 for search strategy). 
To identify English-language publications of approved DMTs for RRMS, the following databases were searched from inception until December 2016: EMBASE, MEDLINE, MEDLINE In-process, and Cochrane Central Register of Controlled Trials (CENTRAL). Congress abstracts published from 2014-2016 were identified via searches of relevant conference websites (Academy of Managed Care Pharmacy [AMCP], American Academy of Neurology [AAN], Americas Committee for Treatment and Research in Multiple Sclerosis [ACTRIMS], American Neurological Association [ANA], Consortium of Multiple Sclerosis Centers [CMSC], European Academy of Neurology [EAN], European Committee for Treatment and Research in Multiple Sclerosis [ECTRIMS], European Federation of Neurological Societies [EFNS], and International Society of Pharmacoeconomics and Outcomes Research [ISPOR]). A supplementary grey literature search was conducted to identify published and unpublished data from databases including the North American Research Committee on Multiple Sclerosis (NARCOMS) and the Multiple Sclerosis registry database (MSBase). The reference lists of the publications identified during the search process were also examined for studies meeting the inclusion criteria of the review.

\section{Study selection and data extraction}

Studies retrieved through the literature search were screened for inclusion in the review based on pre-defined criteria. The patient population consisted of adults with clinically diagnosed RRMS, but not clinically isolated syndrome or non-relapsing forms of secondary progressive MS. Studies that included other MS subtypes were only included if $>80 \%$ of the population had RRMS. In terms of treatment history, studies must have included follow-up of patients for at least 2 years. There were no restrictions based on the number of prior treatments. All types of cross-sectional and longitudinal studies (prospective or retrospective) were eligible for inclusion in the review, including long-term extensions of RCTs, case-control studies, analyses of hospital records/databases, and registry trials. Eligible studies had to include a licensed dose of a DMT (i.e., interferon beta [IFN $\beta$-1a, IFN $\beta$-1b, pegylated (PEG)-IFN $\beta-1 a$ ], glatiramer acetate, natalizumab, fingolimod, teriflunomide, dimethyl fumarate, alemtuzumab, daclizumab [which was voluntarily withdrawn from the worldwide market in 2018], cladribine tablets, or ocrelizumab) as defined by regulatory agencies. Studies that evaluated both licensed and unlicensed doses of DMTs and did not provide subgroup data for the approved dose were only included if $>80 \%$ of the study population received the licensed dose.

The first and second screenings of publications were undertaken by a single reviewer and verified by an independent reviewer. Data were extracted from the included studies for a pre-specified list of outcomes (Supplementary Table 2) by a single reviewer and validated by an independent reviewer.

\section{Results}

\section{Studies identified}

A total of 82 studies (based on 153 publications) met the initial pre-specified inclusion/exclusion criteria of the SLR (Figure 1). Fifteen were long-term extension studies of RCTs and the remaining 67 were observational studies (38 prospective, 20 retrospective, and 9 unspecified). Patient populations included both treatment-naïve and pre-treated patients. Of the 82 studies included in the initial review, many $(>80 \%$ ) did not report discontinuation rates as a key objective. The proportion of patients discontinuing treatment appeared to vary markedly among agents and studies; the wide variety of study designs, objectives and settings made interpretation of this large and disparate body of evidence difficult. Of note, reasons for discontinuation were largely consistent across studies, with lack of efficacy and adverse events (AES) most commonly cited.

To facilitate synthesis of the information, the analysis focused on studies that had discontinuation as their main objective. Additional selection criteria were applied by a single reviewer and verified by an independent reviewer to exclude studies that did not provide a 'real-world' setting perspective (i.e., RCT long-term extension studies) and studies where the terms "persistence," "discontinuation" or "withdrawal" were not specified in the title or the aims or methods sections. Studies investigating the impact of devices on adherence or persistence were also excluded.

After applying the additional selection criteria, 24 studies (from 37 publications) remained that reported discontinuation rates as a key objective. Data were extracted and analysed using a qualitative, narrative manner; due to the heterogeneity in reporting, no statistical analyses were undertaken. Of these 24 studies, the majority (14 studies) evaluated only injectable treatments, four studies evaluated only intravenous infusion therapies (all natalizumab), two studies evaluated only oral treatments (all fingolimod), and four studies compared DMTs with different modes of administration (Table 1). No studies reporting discontinuation rates for PEG-IFN $\beta$-1a, alemtuzumab, ocrelizumab, daclizumab, or cladribine tablets met the revised inclusion criteria. The following results will focus solely on these 24 studies, including a summary of study characteristics and all-cause treatment discontinuation data in Table 2 and the most common reasons for treatment discontinuation in Table 3. An appraisal of all the studies using the Downs and Black checklist was conducted and the mean total score representing bias was $12.95(\mathrm{SD}=3.38) .^{20}$

\section{Injectable treatments (14 studies)}

Of the 14 studies that investigated only injectable treatments, seven evaluated various injectables, five evaluated only IFN $\beta$, and two evaluated only glatiramer acetate.

\section{Various injectables (seven studies)}

Discontinuation and persistence with injectables (IFN $\beta$ and glatiramer acetate) varied substantially among studies. Median time to discontinuation of injectable treatments was 10.2 years in a cohort of 1,032 patients in Turkey (1996-2005). ${ }^{21}$ In this study of 419 participants who discontinued a subcutaneous or intramuscular injectable treatment, $48 \%$ switched to an oral or a second-line DMT, $50.4 \%$ switched to another injectable, and only $1.7 \%$ remained untreated. A longer duration of treatment persistence on injectables was reported in a single centre study of 1,471 patients in Canada between 1996-2011. Patients were observed over an 18-year period with a mean follow-up time of 6.1 years. $^{22}$ In this study, patients were prescribed either IFN $\beta$ (subcutaneous or intramuscular) or glatiramer acetate (subcutaneous) as their first DMT. Median time to discontinuation of the initial DMT was 8.6 years, and median time to complete discontinuation of all injectable DMTs was 11.1 years. Of 610 patients who discontinued injectables, 331 (54\%) resumed treatment with a DMT and 279 (46\%) remained off treatment. The main reasons for discontinuation were AEs (48\%) and lack of efficacy (34\%). In contrast, a study of 410 Portuguese patients treated with injectables as their first DMT between 2000-2013 found that mean time to discontinuation was relatively short (39.8 months). ${ }^{23}$ After 1 year of treatment, $16.3 \%$ had discontinued, and $50.2 \%$ discontinued during the full study period (mean follow-up 6.1 years). After adjustment for baseline Expanded Disability Status Scale (EDSS), subcutaneous IFN $\beta$-1a (22 $\mu \mathrm{g})$ was associated with significantly lower persistence than intramuscular IFN $\beta$-1a (hazard ratio $[\mathrm{HR}]=0.48, \mathrm{p}=0.006$ ); there were no 
Figure 1: Studies identified for inclusion in the systematic literature review

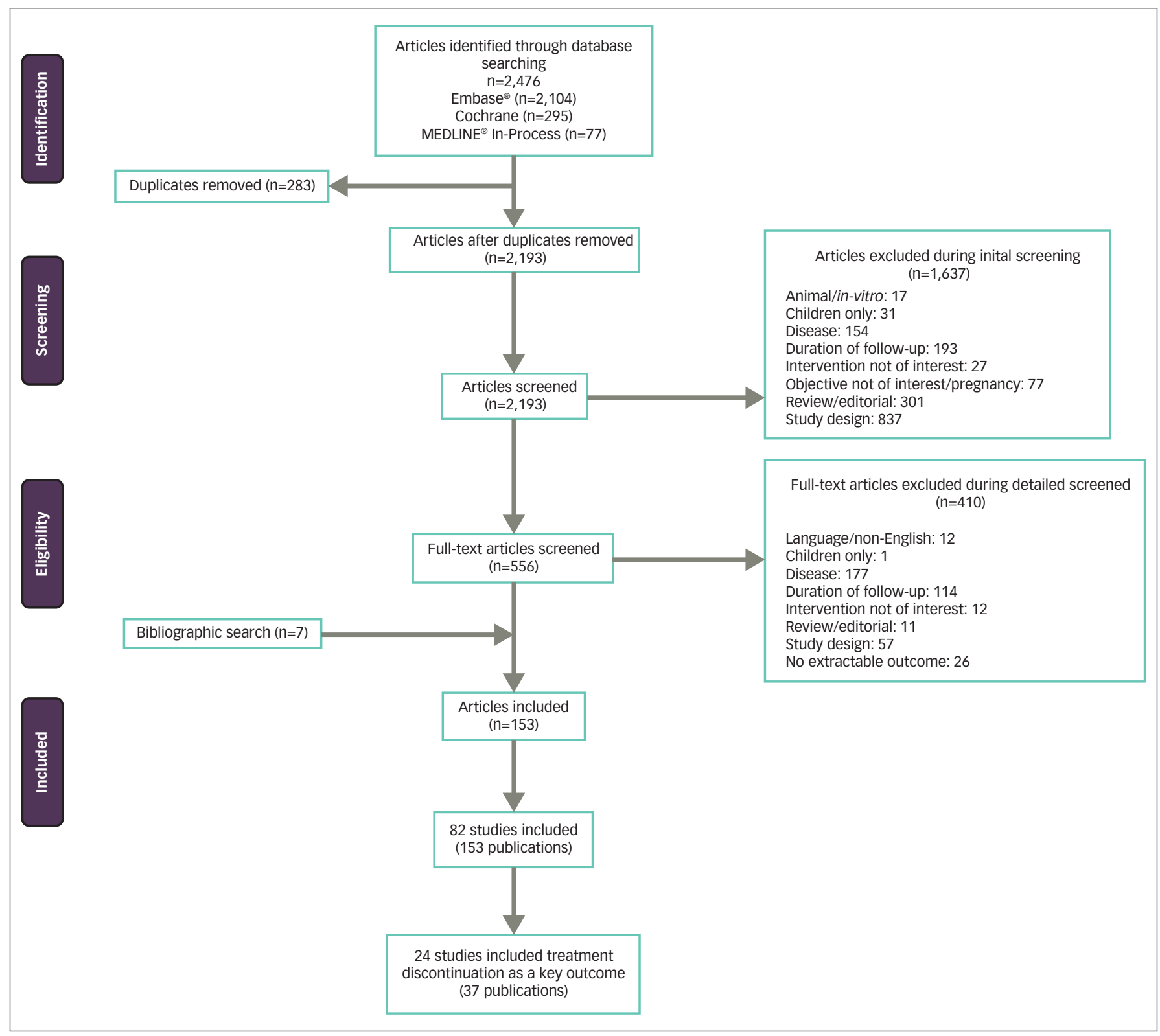

other significant differences in persistence between DMTs. The most common reason for treatment discontinuation for all the DMTs was lack of efficacy (69.4\%), followed by AEs (27.2\%). ${ }^{23}$

A retrospective study of 230 patients from the Innsbruck MS database in Austria investigated outcomes after DMT discontinuation in patients with RRMS and reported a mean time of DMT use of 3.3 years over 5 years of follow-up. ${ }^{11}$ The majority of the study population was using subcutaneous or intramuscular injectables, although 17.8\% were taking other DMTs. As in other studies, AEs were the main reason for discontinuation (45.7\%), although $26.5 \%$ of discontinuations were due to 'stable disease course'. In that study, discontinuing injectables without switching to another DMT was also found to increase the risk of relapse over a 2-year period. A retrospective cohort study (2000-2010) of 128 patients in Italy was conducted to investigate the effect of drug withdrawal on the course of RRMS. Discontinuation of DMT for at least 6 months (46.9\% discontinued over a median follow-up of 108 months) in patients treated with subcutaneous or intramuscular injectables reduced time to relapse compared with patients who persisted with treatment. ${ }^{24}$ Median time to relapse was 31.1 and 85.8 months in the discontinuing and persisting groups, respectively $(p<0.001)$. The main reasons for discontinuation were AEs (63.3\%) and desire for pregnancy (18.3\%); the study excluded patients who discontinued due to lack of efficacy.

The preliminary results of a post-marketing study in Italy in patients with RRMS treated with subcutaneous or intramuscular injectables attending the Lombardia Region's Multiple Sclerosis Centers between January-March 2005 reported a total of 294 patients treated with IFN $\beta$ or glatiramer acetate. ${ }^{25}$ Eighty-seven out of 294 patients (29.6\%) discontinued treatment, most of them within the first three years; no differences were found between therapies. Reasons for treatment discontinuation included persistence of relapses, disability progression, shift to secondary-progressive MS, AES, no consent or loss to followup, and pregnancy. Fifty out of 87 patients (57\%) who discontinued treatment remained treatment-free for at least 1 month; 21 patients (42\%) experienced one or more relapses after discontinuation. Forty-eight patients switched to a second therapeutic agent. 
Table 1: Studies with data on treatment discontinuation as a key outcome

\begin{tabular}{|c|c|c|c|c|c|c|c|c|c|c|}
\hline Study & $\begin{array}{l}\text { IFN } \beta-1 a \\
\text { (Rebif }^{\oplus} \text {; } \\
\text { SC) }\end{array}$ & $\begin{array}{l}\text { IFN } \beta-1 a \\
\text { (Avonex } \\
\text { IM) }\end{array}$ & $\begin{array}{l}\text { IFN } \beta-1 \mathrm{~b} \\
\text { (Betaferon }{ }^{\circledast} \text {; } \\
\text { SC) }\end{array}$ & $\begin{array}{l}\text { Glatiramer } \\
\text { acetate } \\
\text { (SC) }\end{array}$ & $\begin{array}{l}\text { Natalizumab } \\
\text { (IV) }\end{array}$ & $\begin{array}{l}\text { Fingolimod } \\
\text { (oral) }\end{array}$ & $\begin{array}{l}\text { Teriflunomide } \\
\text { (oral) }\end{array}$ & $\begin{array}{l}\text { Dimethyl } \\
\text { fumarate } \\
\text { (oral) }\end{array}$ & $\begin{array}{l}\text { Other - } \\
\text { not } \\
\text { specified }\end{array}$ & $\begin{array}{l}\text { Additional } \\
\text { publications }\end{array}$ \\
\hline
\end{tabular}

\begin{tabular}{|c|c|c|c|c|c|c|c|c|c|c|}
\hline $\begin{array}{l}\text { Bsteh et al. } \\
2016 * 11\end{array}$ & $x$ & $x$ & $x$ & $x$ & & & & & $x$ & \\
\hline $\begin{array}{l}\text { Correia et al. } \\
2016^{23}\end{array}$ & $x$ & $x$ & $x$ & $x$ & & & & & & $\begin{array}{l}\text { Correia et al. } \\
2015^{49}\end{array}$ \\
\hline $\begin{array}{l}\text { Duquette et al. } \\
2016^{41}\end{array}$ & $x$ & $x$ & $x$ & $x$ & $x$ & $x$ & $x$ & $x$ & & $\begin{array}{l}\text { Haddad et al. } 2015^{19} \\
\text { Haddad et al. } 2017^{50} \\
\text { Duquette et al. } 2016^{51}\end{array}$ \\
\hline $\begin{array}{l}\text { Fernández- } \\
\text { Fournier et al. } \\
2015^{33}\end{array}$ & & & & $x$ & & & & & & \\
\hline $\begin{array}{l}\text { Frisell et al. } \\
2015^{40}\end{array}$ & & & & & $x$ & $x$ & & & & $\begin{array}{l}\text { Holmén et al. } 2009^{52} \\
\text { Holmén et al. } 2011^{53} \\
\text { Jonsson et al. } 2013^{54} \\
\text { Matell et al. } 2012^{55} \\
\text { Johansson et al. } 2015^{56}\end{array}$ \\
\hline Hader $2012^{26}$ & $x$ & & $x$ & $x$ & & & & & & \\
\hline He et al. $2015^{42}$ & $x$ & $x$ & $x$ & $x$ & & $x$ & & & & \\
\hline $\begin{array}{l}\text { Jokubaitis et al. } \\
2013^{43}\end{array}$ & $x$ & $x$ & $x$ & $x$ & $x$ & & & & & Jokubaitis et al. $2011^{57}$ \\
\hline $\begin{array}{l}\text { Lapierre et al. } \\
2016^{38}\end{array}$ & & & & & & $x$ & & & & \\
\hline $\begin{array}{l}\text { Lebrun-Frenay } \\
\text { et al. } 2016^{32}\end{array}$ & & & & $x$ & & & & & & Clanet et al. $2015^{58}$ \\
\hline $\begin{array}{l}\text { Lonergan et al. } \\
2013^{35}\end{array}$ & & & & & $x$ & & & & & \\
\hline Lus et al. $2015^{24}$ & $x$ & $x$ & $x$ & $x$ & & & & & & \\
\hline $\begin{array}{l}\text { Mesaroš et al. } \\
2012^{29}\end{array}$ & $x$ & & $x$ & & & & & & & \\
\hline $\begin{array}{l}\text { Midaglia et al. } \\
2016^{36}\end{array}$ & & & & & $x$ & & & & & \\
\hline $\begin{array}{l}\text { Milanese et al. } \\
2005^{25}\end{array}$ & $x$ & $x$ & $x$ & $x$ & & & & & & \\
\hline $\begin{array}{l}\text { Moccia et al. } \\
2016^{27}\end{array}$ & $x$ & $x$ & $x$ & & & & & & & \\
\hline $\begin{array}{l}\text { Nieves Sedano } \\
\text { et al. } 2016^{39}\end{array}$ & & & & & & $x$ & & & & \\
\hline $\begin{array}{l}\text { Özakbas et al. } \\
2019^{21}\end{array}$ & $x$ & $x$ & $x$ & $x$ & & & & & & \\
\hline $\begin{array}{l}\text { Portaccio et al. } \\
2008^{30}\end{array}$ & $x$ & $x$ & $x$ & & & & & & & \\
\hline $\begin{array}{l}\text { Prosperini et al. } \\
2015^{37}\end{array}$ & & & & & $x$ & & & & & \\
\hline $\begin{array}{l}\text { Siger et al. } \\
2011^{28}\end{array}$ & $x$ & $x$ & $x$ & & & & & & & \\
\hline $\begin{array}{l}\text { Toncev et al. } \\
2011^{31}\end{array}$ & $x$ & $x$ & $x$ & & & & & & & \\
\hline Tur et al. $2012^{34}$ & & & & & $x$ & & & & & \\
\hline $\begin{array}{l}\text { Zhornitsky et al. } \\
2015^{22}\end{array}$ & $x$ & $x$ & $x$ & $x$ & & & & & & \\
\hline
\end{tabular}

IFN $\beta$ = interferon beta; $I M=$ intramuscular injection; $I V=$ intravenous infusion; $S C=$ subcutaneous injection

*Treatments described as disease-modifying therapies, including IFN $\beta$ (67.0\%), glatiramer acetate (15.2\%) or other drugs (17.8\%) 
Table 2: Study characteristics and all-cause treatment discontinuation

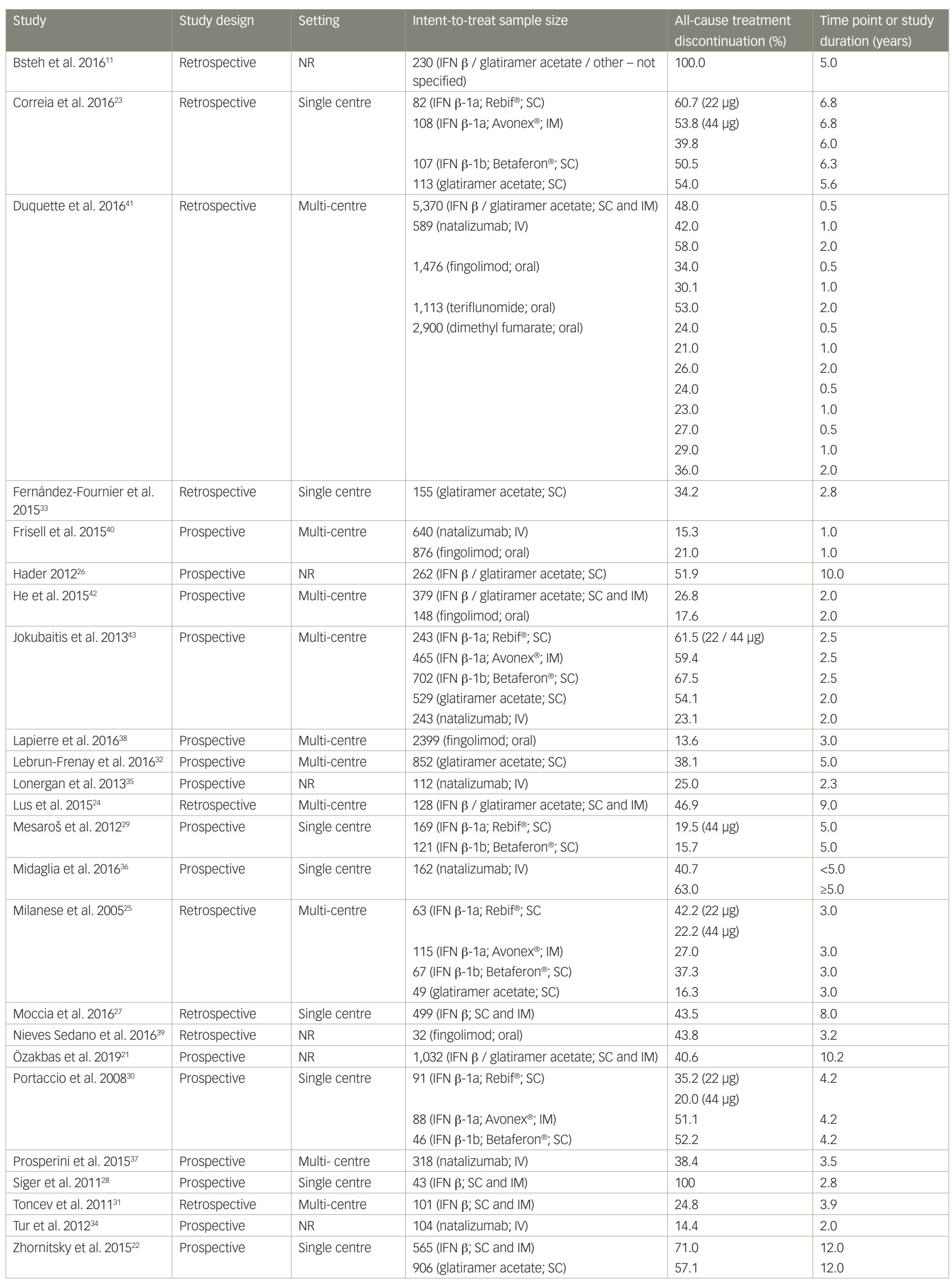

IFN $\beta=$ interferon beta; IM = intramuscular injection; $I V=$ intravenous infusion; $N R=$ not reported; $S C=$ subcutaneous injection 
Table 3: Most common reasons for treatment discontinuation by disease-modifying therapy

\begin{tabular}{|c|c|c|c|c|}
\hline Disease-modifying therapy & $\begin{array}{l}\text { Route of } \\
\text { administration }\end{array}$ & \multicolumn{3}{|c|}{ Reasons identified* (\% of cases) } \\
\hline IFN $\beta$-1a (Rebif $\left.{ }^{\circledR}\right)^{23,25,29,30,43}$ & SC & AE (21-65\%) & Lack of efficacy (4-76\%) & Other (3-42\%) \\
\hline IFN $\beta$-1b (Betaferon $\left.{ }^{\circledR}\right)^{23,25,30,43}$ & SC & AE (11-100\%) & Lack of efficacy (6-100\%) & Lost to follow-up (28\%) \\
\hline Glatiramer acetate $22,23,25,32,33,43$ & SC & AE (14-67\%) & Lack of efficacy (9-75\%) & Patient request (25-42\%) \\
\hline Fingolimod ${ }^{38-40}$ & Oral & AE (27-71\%) & Lack of efficacy (9-50\%) & Withdrawal of consent (4-39\%) \\
\hline
\end{tabular}

*Ranking based on reasons for discontinuation per study (median \% of all-cause treatment discontinuation)

$A E=$ adverse event; IFN $\beta=$ interferon beta; IM = intramuscular injection; IV = intravenous infusion; JCV = John Cunningham virus; PML = progressive multifocal

leukoencephalopathy; $\mathrm{SC}=$ subcutaneous injection

A prospective open-label cohort study was conducted in Saskatchewan Canada of 262 patients treated with subcutaneous IFN $\beta$ or glatiramer acetate who were followed for up to 12 years. ${ }^{26}$ At 10 years, 72 patients (27.5\%) remained on their initial prescription. In that study, 121 patients (48.1\%) had remained on a DMT (including 54 switches), and 136 patients (51.9\%) had discontinued treatment at a mean duration of 4.6 years.

\section{Beta-interferons (five studies)}

In comparative studies of IFN $\beta$, the proportion of patients discontinuing treatment varied markedly both within and across preparations. A retrospective study of 499 patients in Italy (2001-2010) reported that 217 patients (43.5\%) discontinued subcutaneous or intramuscular IFN $\beta$ treatment during the follow-up period. ${ }^{27}$ The study also examined predictors of IFN $\beta$ discontinuation in newly diagnosed patients with RRMS and found that most of the factors associated with discontinuation were demographic or disease-related. Although many of the factors associated with IFN $\beta$ discontinuation were considered by the authors as non-modifiable, the authors nevertheless concluded that these should be taken into account when prescribing the patient's first DMT. Another study examined the effect of discontinuing subcutaneous or intramuscular IFN $\beta$ after 2 years of treatment, in line with Polish reimbursement criteria at the time of the study. ${ }^{28}$ The study analysed 43 patients with high pre-treatment disease activity who responded well to IFN $\beta$ during treatment. Discontinuing IFN $\beta$ after a fixed duration of 2 years was found to result in a rapid return to pre-treatment levels of disease activity. Within approximately 34 months post-treatment, 28 patients (65\%) experienced at least one severe relapse, with eight patients experiencing a relapse within 30 days post-IFN $\beta$ discontinuation, indicating that IFN $\beta$ did not induce a prolonged remission in patients with highly active RRMS. ${ }^{28}$

Although Moccia and colleagues (2016) found the use of subcutaneous IFN $\beta$ - $1 \mathrm{~b}$ to be associated with a $50 \%$ increase in the risk of discontinuation compared with high-dose, subcutaneous IFN $\beta-1 a(H R=1.506 ; p=0.019),{ }^{27}$ other studies of IFN $\beta$ treatment have found no significant differences in persistence or discontinuation across different IFN $\beta$ products. .9,30 For $^{2}$ example, a prospective cohort of 290 patients initiating treatment with a subcutaneous IFN $\beta$ in Serbia from 2004-2009 were followed over a 6 -year period. ${ }^{29}$ Overall, $18 \%$ of patients stopped the treatment with the main reason for treatment discontinuation being lack of efficacy (54\%); $21 \%$ of patients stopped therapy because of pregnancy and $17 \%$ because of AEs. A prospective study in Italy assessed the proportion of discontinuation and reasons for drop-outs in patients treated with subcutaneous or intramuscular IFN $\beta^{30}$ Overall, $46 \%$ of 225 patients suspended therapy, $29 \%$ due to lack of efficacy and $15 \%$ because of AEs. The majority of patients who suspended IFN $\beta$ treatment were switched to another DMT. Finally, another study from Serbia (2004-2011) reported that 25 of the 101 patients treated with a subcutaneous or intramuscular IFN $\beta$ discontinued after a mean follow-up of 3.9 years. ${ }^{31}$ Lack of efficacy was the main reason for discontinuation (38\%) followed by AEs (27\%), moving (15\%), and pregnancy (12\%).

\section{Glatiramer acetate (two studies)}

Two studies reported treatment discontinuation outcomes only for subcutaneous injection with glatiramer acetate. In a large cohort from the French national Copaxone ${ }^{\circledR}$ registry of patients starting treatment between $2005-2008$ ( $N=852), 38.1 \%$ of patients had discontinued glatiramer acetate and remained in the study after 5 years of follow-up. ${ }^{32}$ Patients who discontinued due to AEs had a shorter time to discontinuation than those who discontinued for reasons due to lack of efficacy, with half of the patient cohort receiving no further treatment after discontinuing glatiramer acetate. In a smaller retrospective single centre study (2004-2013) from Spain ( $N=155)$, $34.2 \%$ of patients discontinued glatiramer acetate treatment during an average of 2.8 years of follow-up, most commonly due to lack of efficacy (17.4\%) and injection-site reactions (12.9\%). ${ }^{33}$ Treatment-naïve patients had a 2.8 -fold greater risk of discontinuation than those who had previously been treated with IFN $\beta$.

\section{Infusion therapies (four studies)}

All four studies that investigated only intravenous infusion therapies included natalizumab, with no studies evaluating alemtuzumab or ocrelizumab. Studies of natalizumab focused on the risk-benefit of discontinuation in the context of progressive multifocal leukoencephalopathy (PML). PML risk appeared to influence treatment discontinuation, particularly for those at highest risk, ${ }^{34}$ although there was also conflicting evidence. ${ }^{35}$ Tur and colleagues (2012) divided 104 natalizumab-treated patients from Spain into PML risk groups based on known risk factors (John Cunningham virus [JCV] seropositivity, past immunosuppression, and duration of natalizumab treatment), with patients and their physicians then considering whether or not to continue treatment. ${ }^{34}$ Six out of 10 patients (60.0\%) from the highest risk group discontinued, as did nine of 38 patients (23.7\%) from the second-highest group. There were no discontinuations in the intermediate and low risk groups. In addition to PML risk, clinical outcomes and individual physicians' beliefs were reported to have played a role in decision-making. Another study from Spain found that $40.7 \%$ of 162 patients who could have received natalizumab for 5 years discontinued before this time point, including $23.5 \%$ due to risk of PML. ${ }^{36}$ In contrast, a study of 112 patients from Ireland found $25.0 \%$ discontinued natalizumab treatment overall, with explanation of their positive JCV antibody status having little influence on patients' decisions on whether or not to continue treatment..$^{35}$ 
A multicentre prospective study in Italy followed patients who had received natalizumab for at least 2 years without worsening disability. ${ }^{37}$ Patients who discontinued natalizumab due to AEs and those with baseline EDSS $\geq 6$ were excluded. Of 318 patients, $61.6 \%$ remained on treatment after 6 years of follow-up and $38.4 \%$ had discontinued because of concerns about PML risk (after a median duration of 3.5 years); $90 \%$ of patients who discontinued natalizumab switched to another DMT.

\section{Oral treatments (two studies)}

The two studies that investigated only oral treatments both evaluated fingolimod; no studies evaluating dimethyl fumarate, cladribine tablets or teriflunomide were identified. An analysis of data from the Gilenya Go Program ${ }^{\mathrm{TM}}$ in Canada, which captured data from 2,399 patients treated with fingolimod between 2011-2014, reported persistence rates with fingolimod of $80.7 \%, 76.6 \%$ and $76.0 \%$ at 12,24 and 30 months, respectively. ${ }^{38}$ Among the 327 patients (13.6\%) who discontinued by 3 years, $59.8 \%$ was due to AES, followed by patient or physician request. Discontinuation due to lack of efficacy was very low $-1.3 \%$ at 3 years (accounting for $9.3 \%$ of all discontinuations). ${ }^{38}$ In contrast, a small retrospective spanish study of electronic medical records (2011-2015) reported high levels of discontinuation due to lack of efficacy. ${ }^{39}$ Fingolimod was most frequently prescribed as the third agent and $43.8 \%$ of patients discontinued with a mean treatment duration of 37.8 months. Reasons leading to discontinuation included unsatisfactory therapeutic response (35.7\%), AEs (42.9\%), pregnancy (14.3\%), and other causes (7.1\%).

\section{Treatments with different administration modes (four studies)}

A total of four studies compared discontinuation across treatments with different modes of administration. A study using the IMSE drug monitoring registry in Sweden analysed data for 640 and 876 patients who initiated treatment with natalizumab infusion or oral fingolimod, respectively, between 2011-2013.40 Of patients initiating fingolimod, $44 \%$ had previously been treated with natalizumab. Persistence at 1 year was $87 \%$ for natalizumab, $83 \%$ for patients on fingolimod who had not previously received natalizumab, and $76 \%$ for those on fingolimod who had previously received natalizumab. Fingolimod was associated with significantly higher discontinuation due to AEs (9\% and $12 \%$ for natalizumab-naïve and experienced, respectively) compared with natalizumab (3\%). A Canadian claims database analysis of 11,448 patients discontinuing treatment between 2011-2015 across various DMTs reported that over 24 months, discontinuation rates were lowest with oral fingolimod. Patients on fingolimod had lower discontinuation after 6,12 and 24 month periods (24\%, 21\%, and $26 \%$, respectively) than those on subcutaneous or intramuscular injectable therapies (48\%, $42 \%$, and $58 \%)$, monthly infusions with natalizumab (34\%, 30\%, and $53 \%$ ), or oral therapy with dimethyl fumarate $(27 \%, 29 \%$, and $36 \%) .{ }^{41}$

Based on data from the international MSBase database (1996-2014), switching from subcutaneous or intramuscular injectables to oral fingolimod after on-treatment disease activity was found to be associated with greater persistence on fingolimod than switching to another injectable $(\mathrm{HR}=0.55 ; \mathrm{p}=0.04){ }^{42}$ However, this observation was not replicated in any of the sensitivity analyses and the authors noted that these results should be interpreted with caution. The reasons for switching therapy were not reported..$^{42}$ Another study utilising the MSBase registry, this time in Australian patients, concluded that treatment persistence on both injectables and natalizumab infusion was brief. ${ }^{43}$ At the time of publication, subcutaneous glatiramer acetate, subcutaneous or intramuscular IFN $\beta$, and natalizumab could all be used as initial treatment in Australia, with no differences in eligibility criteria, and switching among these agents was common. Median persistence with initial and subsequent treatment was comparable: 2.5 years on the first treatment and 2.3 years on subsequent treatment(s). Glatiramer acetate was associated with shorter persistence than IFN $\beta$. While natalizumab was a rare first choice of DMT, for subsequent DMTs, patients persisted longer with natalizumab than with injectables. The overall annualised rate of switch or cessation was $9.5-12.5 \%$ for IFN $\beta, 11.6 \%$ for glatiramer acetate and $4.4 \%$ for natalizumab. AEs were the most frequent reason for discontinuation for all DMTs.

\section{Discussion}

The objectives of this SLR were to summarise and evaluate the rates and reasons for discontinuation of DMTs in patients with RRMS. Our SLR identified a total of 24 studies based on 37 publications which spanned the past two decades and included most injectables, infusions, and oral DMTs routinely prescribed by clinicians in current clinical practice. Over the last 20 years, the MS treatment landscape has undergone a significant change with the introduction of DMTs with novel modes of action, routes of administration, and dosing schemes. The most significant impact of newer therapies was, not only increased efficacy, but a change in the safety profile. This has meant that some clinicians and patients appear willing to take on more safety risks for an increase in efficacy, particularly if this means slowing disability progression. ${ }^{44}$

None of the studies included in our review assessed discontinuation rates with PEG-IFN $\beta$-1a, alemtuzumab, ocrelizumab, or cladribine tablets. Some of the reasons for discontinuation may, in part, be addressed by these newer DMTs. From the included studies, general trends and reasons for discontinuation were observed and the reasons for discontinuation were largely consistent across studies, with lack of efficacy and AEs the most commonly cited, followed by pregnancy. The majority of studies investigated discontinuations based on the use of injectables. Unwillingness to inject did not seem to be an important reason for discontinuing treatment, while discontinuing injectables was found to result in a rapid return to pretreatment levels of disease activity, indicating that injectable therapies did not induce a prolonged remission in patients with highly active RRMS..$^{24,45}$ Long persistence with injectables may partly reflect the accumulated experience with these therapeutic options wherein until recently, few other treatments were available. It should also be noted that the introduction of DMTs with less frequent dosing regimens, from daily and weekly dosing to bi-monthly, monthly, and annual dosing, may also impact discontinuation rates and reasons for discontinuing and/or switching. Further studies which reflect the diversity of the contemporary treatment landscape will allow analysis to identify if there is a change in the discontinuation rates with the newer generation of infusions and orals. The current review was limited by the paucity of comprehensive data with long observation periods in real-world settings.

Studies of natalizumab also reported low discontinuation rates due to AEs. The risk of PML was a concern among patients and influenced decisions to initiate or discontinue treatment with natalizumab. Other reviews of DMTs have also discussed the importance of risk stratification for PML for decision-making regarding the initiation or discontinuation of natalizumab.

To the best of our knowledge, this is the first SLR that investigated rates and reasons of discontinuation of DMTs in patients with RRMS. A review of tolerability and adherence of DMTs reported that adherence and persistence with therapy is a problem, with $>25 \%$ of patients discontinuing therapy within 1-2 years. ${ }^{44,46}$ With the availability of 
new infusion and oral treatments, which have differing dosing and risk/benefit profiles, it will be important to continue to investigate if there are real-world changes in discontinuation of treatments for RRMS.

As a limitation of the current review, it should be noted that while the focus was to identify treatment discontinuation and the reasons, the heterogeneity of studies with respect to study design, objectives, settings, and analyses was a barrier in conducting meaningful comparisons across the studies and made interpretation of this large and disparate body of evidence difficult. For example, some studies excluded patients who restarted a DMT within 6 months or those who discontinued treatment due to a lack of efficacy, whereas other studies included all causes of discontinuation and had different definitions of discontinuation in terms of the treatment-free period. A further limitation is that the current review excluded studies that specifically examined the impact of injection devices, which have been shown to improve treatment adherence for patients with RRMS. ${ }^{47}$ It should be noted that the generalisability of the studies reviewed may be restricted by geographic factors that could have impacted results. For instance, in some countries where there is ample reimbursement it may be easier to switch therapy. Finally, recent work has found that at 12 months, $21.9 \%$ of dimethyl fumarate patients and $23.6 \%$ of teriflunomide patients had discontinued therapy. ${ }^{48}$

\section{Conclusions}

A limited number of studies have specifically investigated treatment discontinuation in RRMS. Particularly with the introduction of new treatment options, this is an important consideration when selecting initial and follow-on DMT. The most common reasons for discontinuing treatment were AEs, lack of efficacy, and pregnancy, highlighting the importance of considering the risk/benefit profile of DMTs when determining the appropriate DMT. Further investigation and analysis, particularly around newer oral agents, will be important to provide clearer insights to ultimately inform the choice of treatment and improve outcomes for patients with RRMS.
1. Compston A, Coles A. Multiple sclerosis. Lancet. 2008;372:1502-17.

2. Luessi F, Siffrin V, Zipp F. Neurodegeneration in multiple sclerosis: novel treatment strategies. Expert Rev Neurother. 2012;12:1061-77

3. Noseworthy JH, Lucchinetti C, Rodriguez M, Weinshenker BG. Multiple sclerosis. N Eng/ J Med. 2000;343:938-52.

4. Kamm CP, Uitdehaag BM, Polman CH. Multiple sclerosis: current knowledge and future outlook. Eur Neurol. 2014;72:132-41.

5. Jongen PJ. Health-Related quality of life in patients with multiple sclerosis: Impact of disease-modifying drugs. CNS Drugs. 2017;31:585-602.

6. GBD 2015 Neurological Disorders Collaborator Group. Global, regional, and national burden of neurological disorders during 1990-2015: a systematic analysis for the Global Burden of Disease Study 2015. Lancet Neurol. 2017;16:877-97.

7. Farber RS, Sand IK. Optimizing the initial choice and timing of therapy in relapsing-remitting multiple sclerosis. Ther Adv Neurol Disord. 2015;8:212-32

8. Giovannoni G, Butzkueven H, Dhib-Jalbut S, et al. Brain health: time matters in multiple sclerosis. Mult Scler Relat Disord. 2016;9:S5-S48.

9. Johnson KM, Zhou H, Lin F, et al. Real-world adherence and persistence to oral disease-modifying therapies in multiple sclerosis patients over 1 year. I Manag Care Spec Pharm. 2017;23:844-52.

10. Bsteh $G$, Ehling R, Lutterotti $A$, et al. Long term clinical prognostic factors in relapsing-remitting multiple sclerosis: insights from a 10-year observational study. PLOS One. 2016;11:e0158978.

11. Bsteh $G$, Feige J, Ehling R, et al. Discontinuation of disease-modifying therapies in multiple sclerosis - clinica outcome and prognostic factors. Mult Scler J. 2016;23:1241-8.

12. Kister I, Spelman T, Alroughani R, et al. Discontinuing disease-modifying therapy in MS after a prolonged relapse-free period: a propensity score-matched study. I Neurol Neurosurg Psychiatry Res. 2016;87:1133-7.

13. Vidal-Jordana A, Tintore M, Tur C, et al. Significant clinical worsening after natalizumab withdrawal: predictive factors. Mult Scler. 2015;21:780-5.

14. laffaldano $P$, Viterbo $R G$, Trojano M. Natalizumab discontinuation is associated with a rebound of cognitive impairment in multiple sclerosis patients. J Neurol. 2016:263:1620-5.

15. Menzin J, Caon C, Nichols C, et al. Narrative review of the literature on adherence to disease-modifying therapies among patients with multiple sclerosis. J Manag Care Pharm 2013;19(1 Supp A):S24-S40.

16. Comi $G$, Radaelli M, Soelberg Sørensen P. Evolving concepts in the treatment of relapsing multiple sclerosis. Lancet. 2017;389:1347-56

17. Burks J, Marshall T, Ye X. Adherence to disease-modifying therapies and its impact on relapse, health resource utilization, and costs among patients with multiple sclerosis. Clinicoecon Outcomes Res. 2017:9:251-60

18. Meyniel C, Spelman T, Jokubaitis VG, et al. Country, sex, EDSS change and therapy choice independently predict treatment discontinuation in multiple sclerosis and clinically isolated discontinuation in multiple sclerosis
syndrome. PLOS One. 2012; 7:e38661.

19. Haddad $P$, Duquette $P$, Yeung $M$, et al. Comparison of compliance and discontinuation rates among MS patients treated with fingolimod and other disease-modifying therapies: a Canadian retrospective claims analysis. Value Health 2015; 18:A760

20. Downs SH, Black N. The feasibility of creating a checklist for the assessment of the methodological quality both of randomised and non-randomised studies of health care interventions. J Epidemiol Community Health. 1998;52:377-84.

21. Özakbas S, Piri Cinar B, Oz D, et al. Monthly pulse methylprednisolone therapy is effective in preventing permanent disease progression in secondary progressive multiple sclerosis. Arch Neuropsychiatry. 2019;56:115-8.

22. Zhornitsky S, Greenfield J, Koch MW, et al. Long-term persistence with injectable therapy in relapsing-remitting multiple sclerosis: An 18-year observational cohort study. PLoS One. 2015;10:e0123824.

23. Correia I, Marques IB, Sousa M, et al. Predictors of first-line treatment persistence in a Portuguese cohort of relapsing. reatment pulsting multiple sclerosis. J Clin Neurosci. 2016:33:73-8

24. Lus G, Signoriello E, Maniscalco GT, et al. Treatment withdrawal in relapsing-remitting multiple sclerosis: a retrospective cohort study. Eur J Neurol. 2015;23:489-93.

25. Milanese C, Beghi E, Giordano L, et al. A post-marketing study on immunomodulating treatments for relapsing-remitting multiple sclerosis in Lombardia: preliminary results. Neurol Sci. 2005;26(Suppl 4):S171-3.

26. Hader W. The disability outcomes of disease-modifying drugs in multiple sclerosis. Neurology. 2012;78(Meeting Abstracts 1):P01.152.

27. Moccia M, Palladino R, Carotenuto A, et al. Predictors of longterm interferon discontinuation in newly diagnosed relapsing multiple sclerosis. Mult Scler Relat Disord. 2016;10:90-6.

28. Siger M, Durko A, Nicpan A, et al. Discontinuation of interferon beta therapy in multiple sclerosis patients with high pre-treatment disease activity leads to prompt return to previous disease activity. J Neurol Sci. 2011;303:50-2.

29. Mesaroš Š, Stojsavljević N, Duimović-Bašuroski I, et al. Long-term adherence to interferon-beta treatment in a cohort of RRMS patients in Belgrade, Serbia. Clin Neurol Neurosurg. 2012;114:1145-8

30. Portaccio E, Zipoli V, Siracusa G, et al. Long-term adherence to interferon $\beta$ therapy in relapsing-remitting multiple sclerosis. Eur Neurol. 2008;59:131-5.

31. Toncev G, Miletic Drakulic S, Knezevic Z, et al. Prevalence of multiple sclerosis in the Serbian district Sumadija. Neuroepidemiology. 2011;37:102-6.

32. Lebrun-Frenay C, Moulignier A, Pierrot-Deseilligny C, et al. Determinants of treatment persistence with glatiramer acetate in the French national Copaxone registry. Mult scler. 2016:22:331-2.

33. Fernández-Fournier M, Tallón-Barranco A, Chamorro B, et al. Differential glatiramer acetate treatment persistence in treatment-naive patients compared to patients previously treated with interferon. BMC Neurol. 2015;15:141.

34. Tur C, Tintoré M, Vidal-Jordana A, et al. Natalizumab discontinuation after PML risk stratification: outcome from shared and informed decision. Mult Scler. 2012;18:1193-6.

35. Lonergan R, Kinsella K, Kelly S, et al. Does JCV antibody positivity encourage cessation of natalizumab therapy in multiple sclerosis? J Neurol Neurosurg Psychiatr. 2013;84:e2.92-e2.

36. Midaglia L, Vidal-Jordana A, Mulero P, et al. Long-term follow-up of patients treated with natalizumab: results from a single centre cohort. Mult Scler. 2016;22(S3):650-1.

37. Prosperini L, Annovazzi P, Capobianco M, et al. Natalizumab discontinuation in patients with multiple sclerosis: profiling risk and benefits at therapeutic crossroads. Mult Scler. 2015;21:1713-22

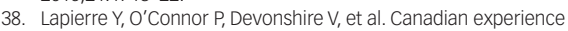
with fingolimod: adherence to treatment and monitoring. Can Neurol Sci. 2016;43:278-83.

39. Nieves Sedano M, Caro Teller JM, Fernandez Redondo $D$, et al. Usage and safety of fingolimod in multiple sclerosis. Int J Clin Pharm. 2016;38:588-9.

40. Frisell T, Forsberg L, Nordin N, et al. Comparative analysis of first-year fingolimod and natalizumab drug discontinuation among Swedish patients with multiple sclerosis. Mult Scler. 2015;22:85-93.
41. Duquette $P$, Rivest $D$, Selchen $D$, et al. A retrospective claims analysis: compliance and discontinuation rates among canadian MS patients treated with disease-modifying therapies-Canadian real world experience. Mult Scler. 2016;22(S3):149-50.

42. He A, Spelman T, Jokubaitis V, et al. Comparison of switch to fingolimod or interferon beta/glatiramer acetate in active multiple sclerosis. JAMA Neurol. 2015;72:405

43. Jokubaitis VG, Spelman T, Lechner-Scott J, et al. The Australian multiple sclerosis (MS) immunotherapy study: a prospective, multicentre study of drug utilisation using the MSBase platform. multicentre study of drug
Plos One 2013:8:e59694.

44. Wingerchuk DM, Carter JL. Multiple sclerosis: current and emerging disease-modifying therapies and treatmen strategies. Mayo Clinic Proc. 2014;89:225-40.

45. Kappos $L$, Kuhle J, Multanen J, et al. Factors influencing long-term outcomes in relapsing-remitting multiple sclerosis: PRISMS-15. J Neurol Neurosurg Psychiatry. 2015;86:1202-7.

46. Giovannoni G, Southam E, Waubant E. Systematic review of disease-modifying therapies to assess unmet needs in multiple sclerosis: tolerability and adherence. Mult Scler. 2012;18:932-46.

47. Lugaresi A. RebiSmart (version 1.5) device for multiple sclerosis treatment delivery and adherence. Expert Opin Drug Deliv. 2013;10:273-83.

48. Lattanzi $S$, Danni M, Taffi $R$, et al. Persistence to oral disease-modifying therapies in multiple sclerosis patients. I Neurol. 2017;264:2325-9.

49. Correia I, Batista s, Marques I, et al. Predictors of first-line treatment persistence in a portuguese cohort of relapsingremitting multiple sclerosis. Eur J Neurol. 2015;22(Suppl 1):821

50. Haddad P, Jobin Gervais K, Schecter R, et al. A retrospective claims analysis on rates of compliance and discontinuation among Canadian multiple sclerosis patients treated with disease-modifying therapies at 6,12 and 24-month periods. Value Health. 2017;20:A719.

51. Duquette P, Yeung M, Schecter R, Haddad P. Compliance and discontinuation rates with Gilenya ${ }^{\varpi}$ and other disease modifying therapies-Canadian real-world experience. Mult Scler. 2016;22:NP20.

52. Holmén C, Piehl F, Hillert J, Olsson T. The immunomodulation and multiple sclerosis epidemiology (IMSE) study: a Swedish nationwide pharmaco- epidemiological and genetic study focused on longterm safety and efficacy of natalizumab. Mult Scler. 2009;15:S129.

53. Holmén C, Piehl F, Hillert J, et al. A Swedish national post-marketing surveillance study of natalizumab treatment in multiple sclerosis. Mult Scler. 2011;17:708-19.

54. Jonsson L, Piehl F, Hillert J, et al. The immunomodulation and multiple sclerosis epidemiology (IMSE) study; a Swedish nationwide pharmacoepidemiological and genetic study focussed on long-term safety and efficacy of natalizumab (Tysabri). Mult Scler. 2013;19:205.

55. Matell H, Sveinsson O, Holmén C, et al. Natalizumab (Tysabri) treatment outcome in terms of safety and efficacy in older patients and/or with longer disease duration - a swedish nationwide long-term follow-up. Mult Scler 2012:18:55-277.

56. Johansson S, Forsberg L, Hillert J, et al. A Swedish nationwide pharmaco-epidemiological and genetic study of the long-term safety and efficacy of natalizumab. Mult Scler. 2015;21:285

57. Jokubaitis VG, Butzkueven $\mathrm{H}$, Spelman T, et al. Treatment utilisation and persistence in the Australian relapsing-remitting MS population: results from the MSBase Registry. Mult Scler. 2011;17:S447-8

58. Clanet M, Moulignier A, Pierrot-Deseilligny $\mathrm{C}$, et al. Five-year follow-up data from the French national Copaxone observatory (P2254). Eur J Neurol. 2015;22:300. 